\title{
Review
}

Journal of Innate

Immunity
J Innate Immun 2011;3:249-257

DOI: $\underline{10.1159 / 000323920}$
Received: October 26, 2010

Accepted after revision: December 7, 2010

Published online: March 22, 2011

\section{Inositol Phospholipid Signaling and the Biology of Natural Killer Cells}

\author{
William G. Kerr ${ }^{a}$ b Francesco Colucci ${ }^{c}$, \\ Departments of a Microbiology and Immunology, and ${ }^{b}$ Pediatrics, SUNY Upstate Medical University, Syracuse, N.Y., \\ USA; ${ }^{C}$ Clinical School, Department of Obstetrics and Gynaecology, and ${ }^{d}$ NIHR Centre for Biomedical Research, \\ University of Cambridge, Cambridge, UK
}

\section{Key Words}

SH2-containing inositol phosphatase 1 .

Phosphoinositide- 3 kinase $\cdot$ Natural killer cells

\begin{abstract}
A family of phosphoinositide-3 kinase (PI3K) isoenzymes catalyzes the production of second messengers that recruit critical regulators of cell growth, survival, proliferation and motility. Conversely, $3^{\prime}$ - (phosphatase and tensin homolog) and $5^{\prime}$-inositol polyphosphatases ( $\mathrm{SH} 2$-containing inositol phosphatases $1 / 2$, SHIP1/2) are recruited to sites of PI3K signaling at the plasma membrane to oppose or, in some cases, to modify and enhance PI3K signaling. A substantial and growing body of literature demonstrates that these enzymes which mediate interchange of phosphates on inositol phospholipid species at the plasma membrane have prominent roles in natural killer cell biology, including development, effector functions and trafficking. Here, we review the salient points of these recent papers with a special emphasis on the role of p1108 and SHIP1 in natural killer cells.
\end{abstract}

Copyright ๑ 2011 S. Karger AG, Basel

\section{KARGER}

Fax +4161306 1234

E-Mail karger@karger.ch

www.karger.com
(C) 2011 S. Karger AG, Basel

Accessible online at: www.karger.com/jin

\section{Fundamental Elements of Inositol Phospholipid Signaling}

Inositol phospholipid-modifying enzymes play an important role in both intracellular trafficking of vesicles and in cell signaling at the plasma membrane. In figure 1, we illustrate the key enzymes and inositol phospholipid species that play a role in cell signals that originate at the intracellular leaflet of the plasma membrane lipid bilayer. The pivotal class of enzymes that promote these signals are the phosphoinositide-3 kinases (PI3K). In response to activating ligands, PI3K are phosphorylated and activated by receptor-associated tyrosine kinases. PI3K in turn phosphorylates the D3 position of nearby $\mathrm{PI}(4,5) \mathrm{P}_{2}$ molecules to yield $\mathrm{PI}(3,4,5) \mathrm{P}_{3}$. The accumulation of $\mathrm{PI}(3,4,5) \mathrm{P}_{3}$ in the plasma membrane signaling provides recruitment sites for signaling proteins containing pleckstrin homology $(\mathrm{PH})$ domains. Signaling proteins that can associate with $\mathrm{PI}(3,4,5) \mathrm{P}_{3}$ include PDK1, Akt, Grp1, DAPP and Tec family kinases (e.g. Btk). These downstream mediators of PI3K signaling promote a divergent array of cellular functions including survival, proliferation or effector functions. Phosphatase and tensin homolog (PTEN) can prevent the initiation of these PI3K effector signals by removal of the $\mathrm{D} 3$ phosphate to revert $\mathrm{PI}(3,4,5) \mathrm{P}_{3}$ to $\mathrm{PI}(4,5) \mathrm{P}_{2}$, and thereby, limiting re- 


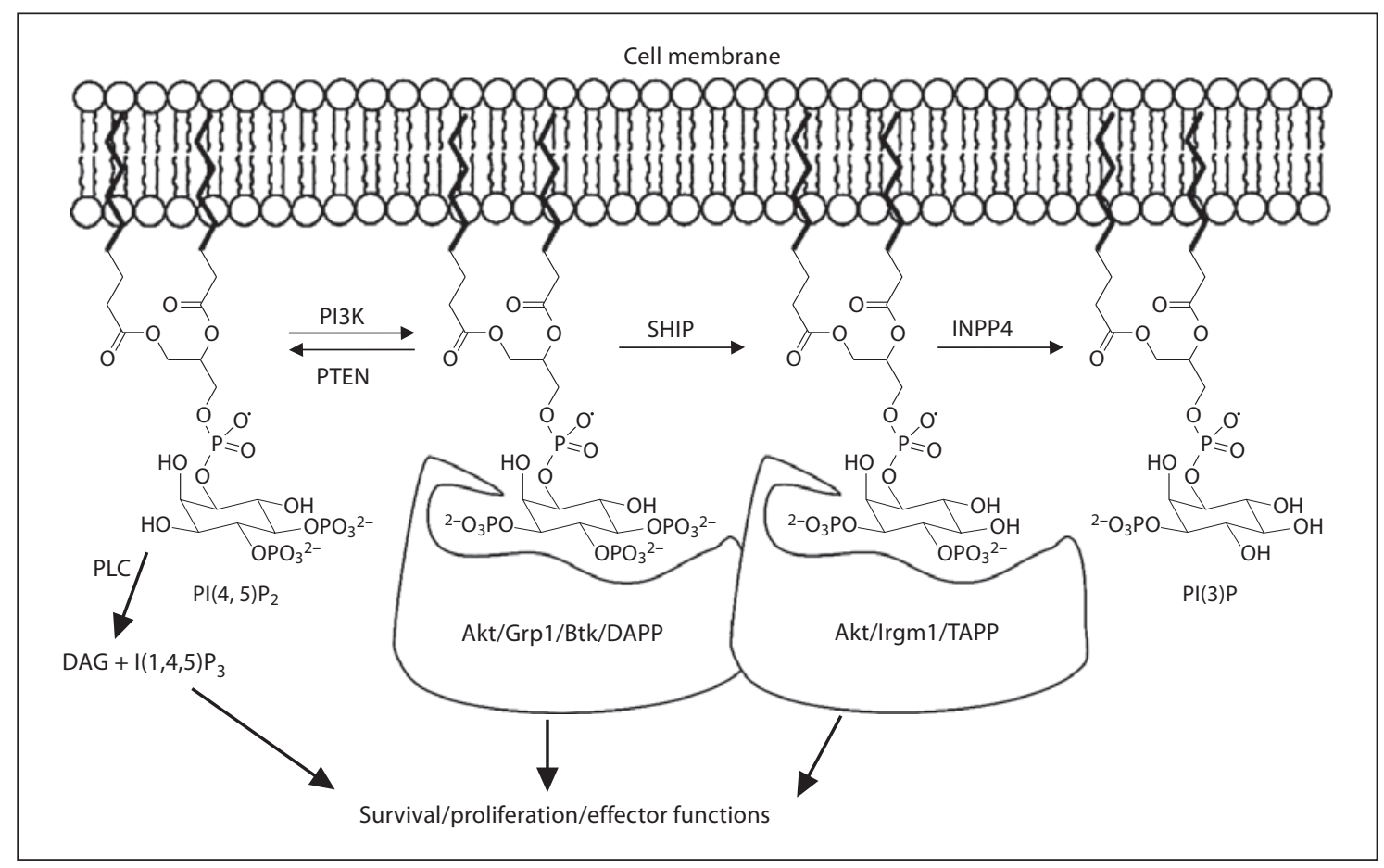

Fig. 1. Plasma membrane-based inositol phospholipids and the enzymes that modify them. PLC = Phospholipase $\mathrm{C} ; \mathrm{DAG}=$ diacylglycerol.

cruitment of these PI3K effectors. In this sense, PTEN opposes the actions of PI3K. Alternatively, $\mathrm{PI}(3,4,5) \mathrm{P}_{3}$ can be converted to $\mathrm{PI}(3,4) \mathrm{P}_{2}$ by $\mathrm{SH} 2$-containing inositol phosphatase 1 (SHIP1) or its paralog SHIP2. This reaction may also extinguish signals from PI3K effectors with $\mathrm{PI}(3,4,5) \mathrm{P}_{3}$ selective $\mathrm{PH}$ domains; however, the SHIP1/2 product, $\mathrm{PI}(3,4) \mathrm{P}_{2}$ also provides recruitment sites for other PI3K effectors that have $\mathrm{PH}$ domains selective for $\mathrm{PI}(3,4) \mathrm{P}_{2}$ (e.g. Akt, Bam32, TAPP1/2, Irgm1), and thus, SHIP1/2 may also in certain contexts promote or modify $\mathrm{PI} 3 \mathrm{~K}$ signals. Thus, depending upon the $\mathrm{PH}$ domain containing effectors of PI3K signaling available for recruitment to a given receptor complex in a cell, SHIP can oppose, enhance or modify PI3K signaling [1]. An activating role for SHIP in PI3K signaling is context dependent, as recently shown in certain blood cell cancers like acute myelogenous leukemia or multiple myeloma where SHIP actually enhances Akt/PKB activation and thus survival of these cancer cells [2]. Recently, a new class of inositol phospholipid signaling enzymes, inositol phospholipid phosphatase 4A (INPP4A) [3] and INPP4B [4], have been shown to preferentially hydrolyze the SHIP product $\mathrm{PI}(3,4) \mathrm{P}_{2}$ to $\mathrm{PI}(3) \mathrm{P}[3,4]$. Loss or mutation of INPP4A or
INPP4B leads to transformation of mouse embryonic fibroblasts and malignant transformation of mammary epithelium, respectively, confirming a promoting role for SHIP1 and SHIP2 in certain cancers [2]. Although $\mathrm{I}(1,4,5) \mathrm{P}_{3}$ is not a membrane-anchored inositol phospholipid, it can also promote cell proliferation, survival or effector functions by increasing intracellular $\mathrm{Ca}^{2+}$ levels following activation of phospholipase $\mathrm{C}$ enzymes by tyrosine kinases activated following their recruitment to $\mathrm{PI}(3,4,5) \mathrm{P}_{3}$ (e.g. Btk). Thus, $\mathrm{Ca}^{2+}$ release, in certain contexts, can be promoted by PI3K and opposed by SHIP [5].

\section{PI3K and SHIP1}

The PI3K pathway is executed by a number of isoenzymes that organize vital functions in all cells. Class I PI3Ks are the most thoroughly studied in mammalian cells and are composed of 4 isoenzymes subdivided into class IA (p110 $\alpha, \mathrm{p} 110 \beta, \mathrm{p} 110 \gamma, \mathrm{p} 110 \delta)$ and class IB (p110 $\gamma)$

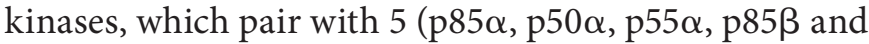
$\mathrm{p} 55 \gamma$ ) and 2 (p101 and p84) PI3K regulatory subunits, respectively. p1 $10 \alpha$ and $\mathrm{p} 110 \beta$ are ubiquitously expressed, 
whereas p110 $\gamma$ and $\mathrm{p} 110 \delta$ are mainly expressed in leukocytes [6]. The 4 PI3K isoenzymes catalyze a biochemical reaction that produces the transient, yet critical, lipid second messenger $\mathrm{PI}(3,4,5) \mathrm{P}_{3}$ that, upon association with the signaling molecules mentioned above, control disparate aspects of cell biology, including growth, survival, metabolism and motility [7] and are prominent pharmacological targets [8-11].

Despite the invariant nature of the final product, accumulating evidence suggests that the 4 p110 isoforms have specific functions [10]. The complexity of the pathway poses, on the one hand, a stimulating intellectual challenge and, on the other, offers the opportunity to intervene therapeutically using small molecule inhibitors that selectively target specific PI3K catalytic isoforms [8]. Small molecule inhibitors of PI3K isoforms have entered clinical trials to treat cancer and inflammatory diseases [8-10], and some of the expected therapeutic effects are through inhibition of leukocyte migration [11]. Therefore, it is important to learn the consequences of the cellspecific inactivation of individual PI3K isoforms on health and disease. The ubiquity of the pathway notwithstanding, tissue-specific expression of some catalytic isoforms creates the possibility of limiting the inhibitory effect of these small molecules to specific cell types and/or target tissues. For example, p110 $\delta$ is preferentially expressed in both normal leukocytes, including natural killer (NK) cells and leukemic cells.

We and others identified a gene currently referred to as SHIP [12-16]. SHIP was isolated by gene trapping of lipopolysaccharide response genes in B-lymphoid cells [12], for its ability to associate with the phosphotyrosine binding domain of Shc $[13,15]$ or the $\mathrm{SH} 3$ domain of Grb2 [14]. The sequence of SHIP indicated that it is likely to play a role in several signal transduction pathways due to its SH2 domain, an inositol 5' -phosphatase domain, polyproline-rich regions for binding to $\mathrm{SH} 3$ domains (PxxP), a C2 domain that can bind to its product $\mathrm{PI}(3,4) \mathrm{P}_{2}, \mathrm{NPXY}$ sequences that can be phosphorylated and associate with phosphotyrosine binding domains and a YIGM motif that can be recognized by the $85-\mathrm{kDa}$ regulatory subunit of PI3K [12-17]. In hematolymphoid cells, SHIP can be recruited to a wide variety of receptor complexes including growth factor receptors $[13,18-23]$ and immune receptors such as FcR $\gamma I I b$, Fc $\gamma$ RIII, Ly49A-C, KLRG1 and 2B4 [24-28]. SHIP is recruited to receptor-associated signaling complexes via adapters (e.g. Shc, Grb2, Dok3), scaffold proteins like Gab1/2 or directly via its $\mathrm{SH} 2$ domain $[13,19-23,29,30]$. After recruitment to the plasma membrane, SHIP can then hydrolyze $\mathrm{PI}(3,4,5) \mathrm{P}_{3}$ and in

NK Cells and Inositol Phospholipid

Signaling so doing attenuate several different PI3K effector pathways. Hydrolysis of $\mathrm{PI}(3,4,5) \mathrm{P}_{3}$ inhibits recruitment of $\mathrm{PH}$ domain-containing kinases like Akt, Btk and phospholipase $\mathrm{C}-\gamma$ to the plasma membrane. By antagonizing the membrane recruitment and activation of these $\mathrm{PH}-$ containing kinases, SHIP limits several different downstream PI3K effectors that control survival, differentiation and/or proliferation. These include downstream cytoplasmic kinases like MAP/ERK [31, 32], JNK/SAPK [33], p38 MAPK [31, 32] and key transcription factors such as nuclear factor- $\kappa B$ [31] and NFAT [5]. Subsequent to the cloning of the $\mathrm{SH} 2$-containing isoform, we identified a stem cell-specific isoform of SHIP, s-SHIP, that lacks the $\mathrm{SH} 2$ domain due to initiation at an intronic promoter between exons 5 and 6 [29]. s-SHIP is biochemically distinct from SHIP in that it is constitutively recruited to the plasma membrane of stem cells in association with Grb2 [29]. Moreover, it is recruited to the plasma membrane in the absence of tyrosine phosphorylation [29].

\section{NK Cells}

The discovery of NK cells was based on their propensity to recognize and spontaneously lyse tumor cells [3436]. Subsequently, an important immunoregulatory role of NK cells was appreciated. NK cells have physiological roles in reproduction and tissue homeostasis and participate in immunological surveillance of cancer and infection. Furthermore, they regulate adaptive immunity and constitute an important histocompatibility barrier, particularly in bone marrow (BM) transplantation. Being endowed with these important recognition systems and instant effector function potential, NK cells have become a prominent immune effector in modern medicine.

The common denominator of the diverse roles of NK cells is probably to be found in a set of genes that encode a wide array of receptors. These receptors interact with other cell surface molecules within the individual, between individuals and across genomes. Examples of the elements recognized by NK cells are self-major histocompatibility complex (MHC)-I antigens, stress-inducible ligands during infections or tumor transformation, donor antigens on tissue grafts, paternal antigens at the maternal-fetal interface and viral products. The nature of these interactions sets the threshold for NK cell activation, which in turn has downstream consequences on innate immunity and adaptive responses. An important aspect of NK cell biology emerging in recent years is that the 
functional versatility of NK cells is the result of subset heterogeneity and appropriate location. NK cells develop primarily in the BM, but development has also been reported in the thymus and lymph nodes. They are distributed throughout all lymphoid tissues, as well as in most organs, including the uterus, liver, lungs, intestine and peritoneum.

\section{Inositol Phospholipid Signaling in NK Cell Development, Homeostasis and Repertoire Formation}

Following the pioneering work of Kanakaraj et al. [37] and Bonnema et al. [38] who first investigated the role of PI3K in NK cell antibody-dependent cell-mediated cytotoxicity and spontaneous cytotoxicity, Jiang et al. [39] elegantly dissected the PI $3 \mathrm{~K} \rightarrow \mathrm{Racl} \rightarrow \mathrm{PAK} 1 \rightarrow$ MEK $\rightarrow$ ERK pathway that leads to degranulation. We uncovered Sykindependent functions in NK cells that were dependent on PI3K [40, 41]. These studies were all performed using general inhibitors of PI3K such as wortmannin and LY2940002. The first studies on lymphocytes of PI3Ktargeted mice was described in 1999 [42, 43], and the first study on NK cells of PI3K-targeted mice was described in 2007 [44]. Five more papers followed shortly after [4549]. These papers, while not always in agreement, have generated a critical mass of information that we review below with the aim of summarizing the common themes revolving around the role of $\mathrm{p} 110 \delta$ in NK cell biology.

Recent advances in NK cell development have highlighted critical stages identifiable by flow cytometry. For example, NK cell maturity is marked by the expression of CD11b and CD27, and p1108 knockin mice have fewer mature $\mathrm{CD}_{11 \mathrm{~b}}{ }^{+} \mathrm{CD} 27^{+} \mathrm{NK}$ cells [48]. The acquisition of $\mathrm{MHC}$ receptors marks an important event in NK cell education $[50,51]$. Signaling through these receptors during development is thought to be a prerequisite for the acquisition of NK cell functional competence under certain conditions [52], while in others, such as mouse cytomegalovirus infection [53] and human melanoma [54], 'uneducated' NK cells are functionally competent. Indeed, mice that are deficient for surface expression of 'licensing' MHC-I ligands exhibit normal NK control of cytomegalovirus infection [55]. Ly49G2+ ${ }^{+} \mathrm{NK}$ cells are underrepresented in $\mathrm{H}-2^{\mathrm{d}} \mathrm{p}^{110 \delta^{-/-}}$mice [45], and Ly49C ${ }^{+} \mathrm{Ly} 49 \mathrm{I}^{+}$ cells are underrepresented in $\mathrm{H}-2^{\mathrm{b}}$ mice $[47,48]$. The abnormalities in the Ly49 repertoire are maintained in BM chimeras [47], suggesting that the acquisition of Ly 49 receptors that bind self-MHC is regulated cell-autono- mously by $\mathrm{p} 110 \delta$. The $\mathrm{p} 110 \gamma$ isoform cooperates with p110 $\delta$ in establishing the Ly 49 repertoire, as suggested by the finding that some Ly49 receptors are downregulated in p110 $\gamma$ knockout $(\mathrm{KO})$ mice $[45,47]$ and that $\mathrm{p} 110 \gamma \delta$ compound $\mathrm{KO}$ mice have a more profound dysregulation of Ly49 expression [47].

Mice lacking SHIP develop lower frequencies of most Ly $49^{+}$NK cells; however, certain Ly49 receptors can be upregulated, particularly in the presence of strong MHCI ligands $[26,56]$. Interestingly, receptors that do not detect the presence of MHC-I ligands, but rather detect non-MHC self-ligands such as CD48, are also impacted in $\mathrm{SHIP}^{-/-} \mathrm{NK}$ cells $[57,58]$. Moreover, T-cell-specific conditional $\mathrm{KO}$ of PTEN, the other negative regulator of the pathway, dysregulates the repertoire of Ly49 receptors in NK T cells. The Ly49 repertoire of NK T cells is restored to the normal pattern in $\mathrm{PTEN}^{-1-}$ mice lacking also p110 $\delta$ and/or p110 $\gamma$ [59]. These data corroborate the notion that PI3K positively regulates the expression of Ly 49 receptors in NK cells and introduce the concept that the negative regulators of the PI3K pathway may oppose this function. Although it appears evident that PI3K positively regulates the frequency of NK cells expressing certain Ly49 receptors, the mechanisms by which this regulation occurs remain to be elucidated, although effects of PI3K and SHIP/PTEN on the relative survival of different Ly $49^{+} \mathrm{NK}$ cell subsets appear to contribute [26]. Although we do not know at what stage of NK cell development p110 $\delta$ plays its unique role, it is likely to be after commitment to NK lineage differentiation, which is marked by the expression of the $\beta$-chain of the interleukin (IL)-2/ IL-15 receptor (i.e. IL2Rb or CD122). Indeed, NK cell precursors are present in normal numbers in $\mathrm{p} 110 \delta$ mutant mice [48].

Development, repertoire and function of NK cells have been analyzed in mice that have targeted mutations in the 5'-proximal promoter and first exon of SHIP [26]. This mutation ablates expression of $\mathrm{SH} 2$-containing SHIP isoforms, but leaves intact the expression of the stem cell-specific SHIP isoform, s-SHIP, which initiates transcription from an internal promoter located between exons 5 and $6[29,60]$. However, we do not detect expression of s-SHIP protein in FACS-purified NK cells [26]. Our genetic analysis demonstrated that the $\mathrm{SH} 2$-containing isoforms of SHIP play a prominent role in NK cell physiology via its participation in signaling pathways that control survival, homeostasis and repertoire diversity in the peripheral NK compartment [26, 56-58]. Despite the altered repertoire of $\mathrm{SHIP}^{-/-} \mathrm{NK}$ cells which includes underrepresentation of most Ly49 receptors, NK cell devel- 
opment appears to be intact as normal or increased numbers of NK cells are present in the spleen of $\mathrm{SHIP}^{-/-}$mice. In fact, rejection of missing self-BM grafts, a unique function of NK cells, is intact in $\mathrm{SHIP}^{-/-}$hosts indicating that a cytolytically mature NK compartment is present [26]. We have observed a small but significant increase in peripheral NK cell numbers in $\mathrm{SHIP}^{-/-}$mice that is due to decreased turnover rather than to increased production of NK cells $[26,56]$. Consistent with this enhanced survival, Akt/PKB expression and phosphorylation are increased in $\mathrm{SHIP}^{-/-} \mathrm{NK}$ cells [26]. 2B4 and Ly49 receptors that detect self-ligands like CD48 and MHC-I, respectively, may promote enhanced peripheral survival of SHIP $^{-/-}$NK cells as both PI3K and SHIP are recruited to these receptors for self-ligands [26, 27]. We have recently provided direct evidence for $2 \mathrm{~B} 4$ providing homeostatic signals for peripheral NK cells as increased splenic NK cell numbers are not observed in $2 \mathrm{~B}^{-/-} \mathrm{SHIP}^{-/-} \mathrm{NK}$ mice [56]. Ly49 receptor interaction with MHC-I ligands may also provide homeostatic signals for murine NK cells [61] that require regulation by SHIP $[26,56]$. Thus, PI3K and SHIP may be recruited to an array of NK receptors for self-ligands in order to control the relative numbers of various NK cell subsets in the periphery [62].

Although SHIP1 has yet to be directly linked to killer cell immunoglobulin-like receptor (KIR) signaling in human NK cells, others have found that antibody cross-linking of an endogenously expressed KIR in the NK3.3 cell line triggers activation of the PI3K/Akt pathway [63]. Because SHIP can oppose PI3K-mediated activation of Akt and is recruited to the KIR analogs expressed by murine NK cells [26], we proposed that human SHIP1 may also oppose PI3K/Akt signals at KIR in human NK cells [26]. In fact, because the immunoglobulin superfamily KIR receptors have greater affinity for their human leukocyte antigen ligands than their murine analogs, C-type lectin Ly49 receptors, we propose a more prominent role for SHIP in human KIR signaling. Combined KIR and human leukocyte antigen transgenic SHIP-deficient mouse models are under development to test this possibility.

\section{Inositol Phospholipid Signaling and the Cytolytic Function of NK Cells}

Five papers published by three independent groups have shown that p1108 is dispensable for NK cell cytotoxicity, as the lysis of lymphoma cells in vitro is only marginally affected or not affected at all $[44,45,47,48]$ and p110 knockin NK cells are capable of rejecting class-I- deficient lymphoma cells when administered at the site of tumor growth [46]. However, Zebedin et al. [49] showed that the lack of $\mathrm{p} 110 \delta$ is associated with reduced degranulation, as measured by CD107 and lysis of a panel of target cells. This study was done using mice with mixed $129 \times \mathrm{C} 57 \mathrm{BL} / 6$ background, whereas the four preceding studies used mice with C57BL background (B6 or B10.D2). NK cells of 129 mice are poorly cytotoxic due to a proximal signaling defect that has yet to be characterized [64]. We propose that the 129 background may con-

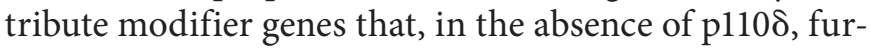
ther exacerbate this cytotoxicity defect. Indeed, we have confirmed that $110 \delta^{-/-} \mathrm{NK}$ cells of mixed $129 \times \mathrm{C} 57 \mathrm{BL} / 6$ are poorly cytotoxic [46] as opposed to p110 $\delta$ mutant NK cells of C57BL/6 and B10.D2 mice, which do mediate cytotoxicity that was found to be not significantly different from that of wild-type mice in three works $[44,45,47]$ and only marginally decreased in one work [48].

Cytotoxicity in the p1108-deficient NK cells may be sustained by compensatory activity of p110 $\gamma$ since simultaneous inactivation of both p110 $\gamma$ and p110 $\delta$ severely reduced lysis of target cells in both NK cells $[45,47]$. Tassi and colleagues [47] demonstrated that catalytically active phospho-ERK 1/2 disappears during target cell conjugation only in the absence of both isoforms, suggesting that, at least within the MAPK transduction pathway, p110 $\gamma$ and p110 $\delta$ are capable of performing similar roles.

SHIP deficiency leads to severe NK repertoire disruptions that compromise certain NK functions [26, 56-58]. Other groups have also begun to observe that SHIP plays a pivotal role in signaling pathways that control human NK cell function in vitro, including inhibition of Fc $\gamma$ RIII signaling such that antibody-dependent cell-mediated cytotoxicity is enhanced when SHIP function was blocked in a human NK cell line by expression of a dominant negative mutant [25]. We have recently identified a critical role for SHIP in 2B4/CD244 signaling in NK cells such that the absence of SHIP expression leads to increased expression of both 2B4/CD244 and SHP1 and to inappropriately high recruitment of SHP1 to $2 \mathrm{~B} 4$ that leads to compromised NK cytolysis via either NKG2D or Ly49H [56-58]. This inhibitory dominance by $2 \mathrm{~B} 4$ occurs in C57BL6 mice with an $\mathrm{H} 2^{\mathrm{b}}$ MHC-I haplotype. Intriguingly, when the SHIP mutation is transferred to an MHCI H $2^{\mathrm{d}}$ homozygous background, 2B4 expression remains dysregulated; however, cytolysis was not compromised and, in fact, was substantially enhanced for MHC-I mismatched targets like $\mathrm{H} 2^{\mathrm{b}+}$ RMA lymphoma cells [56]. One possible explanation for the disparate effects of SHIP deficiency on $\mathrm{H} 2^{\mathrm{b}}$ and $\mathrm{H} 2^{\mathrm{d}}$ haplotype $\mathrm{NK}$ cells is that 
Ly49A, a potent NK licensing or educating receptor for $\mathrm{H} 2^{\mathrm{d}}[52,65]$, is expressed at inappropriately high levels on SHIP $^{-/-} \mathrm{H} 2^{\mathrm{d}} \mathrm{NK}$ cells [56]. The Ly49A receptor is downregulated in the presence of $\mathrm{H}_{2}{ }^{\mathrm{d}}$ ligands [66]. Perhaps SHIP deficiency allows $\mathrm{H} 2{ }^{\mathrm{d}+} \mathrm{NK}$ cells to express the polyspecific MHC-I receptor Ly49A at sufficient levels to enable NK licensing or education of a greater proportion of the NK cell compartment, thus increasing lytic capacity of the NK compartment for MHC-I mismatched tumor targets. This finding and the recent identification of small molecule inhibitors of SHIP1 [2] suggest the possibility that certain human NK or BM donors could be identified that would have greater capacity for killing of residual cancer cells when patients are treated with a SHIP1 inhibitor following allogeneic BM transplantation or after adoptive transfer of donor NK cells. We also find that $\mathrm{H}_{2}{ }^{\mathrm{d}}$ haplotype $\mathrm{SHIP}^{-/-}$mice die with greater frequency and at earlier onset than their $\mathrm{H} 2^{\mathrm{b}}$ counterparts, suggesting the possibility that SHIP is required to maintain NK self-tolerance when a high-affinity MHC-I receptor/ligand pair (e.g. Ly49A-H2D ${ }^{\mathrm{d}}$ ) is present in the genome - a possibility we are currently testing.

\section{Inositol Phospholipid Signaling and NK Cell Migration}

Despite some discrepancies on the role of $\mathrm{p} 110 \delta$ in NK cell-mediated lysis, the role of p110 $\delta$ in NK cell-mediated tumor rejection is clear. Three independent groups have

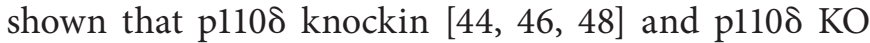
mice [49] fail to reject lymphomas and melanoma cells, unless, as stated above, the mutant NK cells are administered at the site of tumor growth [46]. This observation led us to propose a role for $\mathrm{p} 110 \delta$ in NK cell migration to tumor sites. Indeed, while both p $110 \gamma$ and $110 \delta$ isoforms were required for chemotaxis to SDF-1/CXCL12 and CCL3, only p $110 \delta$ was indispensible for chemotaxis to S1P and CXCL10. In the absence of p1108, NK cell distribution was perturbed and extravasation to tumors failed to occur. While S1P and CXCL10 have been implicated in both distribution and extravasation of NK cells, the scope of p110 involvement in these complex processes remains to be clearly established. However, since the receptors for S1P and CXCL10 are G-protein-coupled S1PR and CXCR3, these findings suggest that G-protein-coupled receptor signaling may not be the exclusive domain of p110 $\gamma$. Besides its role in NK cell migration towards the site of tumor, p110 $\delta$ is also required for the migration of NK cells towards the site of acute inflammation and to- wards the uterus in pregnant mice [44, 46]. Given the prominent role that NK cells are believed to play at the feto-maternal interface, it will be interesting to investigate whether defective PI3K function in uterine NK cells has downstream consequences on reproductive biology.

The role of SHIP in NK cell migration and chemotaxis has not been investigated to date. However, there is evidence for a prominent role of SHIP in the CXCR4-SDF1/ CXCL12 axis. SHIP ${ }^{-/}$myeloid progenitors were found to chemotax to an SDF1/CXCL12 gradient in vitro with greater efficiency [67], suggesting that SHIP opposes the activity of PI3K at CXCR4. However, the situation in vivo is less clear as SDF1/CXCL12 expression in the BM and secondary lymphoid tissues of SHIP-deficient mice is profoundly diminished [68]. Moreover, CXCR4 levels are also downmodulated on $\mathrm{SHIP}^{-/-}$hematopoietic stem cells [69] suggesting that both ligand and receptor expression in the CXCR4-SDF1/CXCL12 axis are impaired in vivo by SHIP deficiency. The mechanism for this downmodulation remains to be defined. Nishio et al. [70] have recently shown that chemotaxis by SHIP-deficient neutrophils is severely impaired, because the cells fail to polarize $\mathrm{PI}(3,4,5) \mathrm{P}_{3}$ to the leading edge of the chemotaxing cell. Thus, it should not be assumed that chemotaxis and migration of SHIP-deficient cells will be enhanced due to unopposed PI3K signaling at chemotactic receptors.

\section{Inositol Phospholipid Signaling in Cytokine Secretion by NK Cells}

NK cells require PI3K signaling for cytokine secretion, but, like cytotoxicity, this requirement is not absolute and can be circumvented by high concentrations of IL- 2 or IL-12/18, thus highlighting various pathways that converge around this key NK cell function. In the absence or in limiting concentrations of stimulatory cytokines, NK cells appear to be more dependent on p110 $\gamma$, thus linking this isoform to the ITAM-based signal to initiate cytokine secretion. However, no deficiency in the phosphorylation of ERK or JNK could be demonstrated in these mutants [47]. The two groups who measured cytokine accumulation in supernatants found it to be severely impaired in the cells with non-functional p110 $[45,48]$. We suggested that since intracellular cytokine levels were indistinguishable from wild type in these cells, the defect must be at the level of the mechanics of secretion. Interestingly, in the kinase-dead $p 110 \delta$ mutants, JNK phosphorylation was reduced in response to signaling through the NKG2D receptor, and the resulting cytokine secretion defect 
could be recapitulated by a JNK inhibitor [48]. In the p110 $\mathrm{KO}$, JNK might have been adequately phosphorylated under the same conditions, perhaps because p110 $\gamma$ can compensate in the complete absence of $\mathrm{p} 110 \delta$, but not in the presence of a non-functional p1108. This discrepancy serves to highlight the differences between the two experimental approaches used by the different groups in dissecting this crucial signaling cascade.

One deficiency that is uniformly observed in SHIPdeficient NK cells, regardless of MHC haplotype or 2B4 genotype, is impaired induction of interferon (IFN)- $\gamma$ after engagement of activating NK receptors, including NK1.1, NKG2D and NKp46 [56]. In fact, IFN- $\gamma$ induction is compromised even in $\mathrm{H}_{2}{ }^{\mathrm{d}}$ haplotype $\mathrm{SHIP}^{-/-} \mathrm{NK}$ cells despite their normal or enhanced cytolytic function. The discordant capacity of $\mathrm{H}_{2}{ }^{\mathrm{d}+} \mathrm{SHIP}^{-/-} \mathrm{NK}$ cells to mediate these two key NK functions indicates that SHIP plays distinctly different signaling roles in control of cytolytic function and IFN- $\gamma$ production. That SHIP is required for IFN- $\gamma$ induction indicates a positive role for SHIP signaling in promoting this NK effector function. This positive signaling role for SHIP may be related to its production of $\mathrm{PI}(3,4) \mathrm{P}_{2}$, and hence, the activation of distal effector kinases selectively recruited by $\mathrm{PI}(3,4) \mathrm{P}_{2}$. Intriguingly, SHIP appears to inhibit IFN- $\gamma$ production by human NK subsets in response to combined stimulation with IL-12 plus IL-18 [71]. The disparity between these findings could simply reflect that the role of SHIP in control of IFN- $\gamma$ production differs depending on the receptor that activates the NK cell. Similarly, IFN- $\gamma$ production by IL12 -stimulated p110 $\delta \mathrm{KO} N K$ cells was greater than that of wild-type NK cells, although no differences were found when $\mathrm{KO}$ and wild-type cells were stimulated through other receptors [47]. However, we [45] and Guo et al. [48] found that cytokine secretion by p110 $\delta$ mutant cells was invariably decreased regardless of the stimulus. The nature of this discrepancy is currently unknown. It has recently been shown that SHIP deficiency leads to a profound Crohn's disease-like disease in mice [72]. An intriguing possibility is that mucosal $\mathrm{SHIP}^{-/-} \mathrm{NK}$ subsets are also defective for the production of IL-22 [73-75], a cytokine critical for the maintenance of an intact intestinal epithelial cell barrier [76].

\section{Acknowledgements}

We thank Nayoung Kim, Aurore Saudemont, Jean-Marc Doisne and Patricia Hunter for constructive discussions, and Klaus Okkenhaug for generously sharing resources. F.C. is funded by grants from the Wellcome Trust, Medical Research Council, Association for International Cancer Research and the Centre for Trophoblast Research. W.G.K. is supported by grants from the US National Institutes of Health (R01 HL72523, RO1 HL085580) and the Paige Arnold Butterfly Run.

\section{References}

1 Kerr WG: Inhibitor and activator: dual functions for SHIP in immunity and cancer. Ann NY Acad Sci 2011;1217:1-17.

2 Brooks R, et al: SHIP1 inhibition increases immunoregulatory capacity and triggers apoptosis of hematopoietic cancer cells. J Immunol 2010;184:3582-3589. DOI: 10.4049/ jimmunol0902844.

3 Ivetac I, et al: Regulation of PI(3)K/Akt signalling and cellular transformation by inositol polyphosphate 4-phosphatase-1. EMBO Rep 2009;10:487-493. DOI: $10.1038 / \mathrm{em}-$ bor200928.

4 Gewinner C, et al: Evidence that inositol polyphosphate 4-phosphatase type II is a tumor suppressor that inhibits PI3K signaling. Cancer Cell 2009;16:115-125. DOI: 10.1016/ jccr200906006.

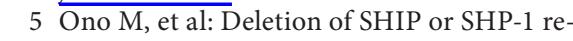
veals two distinct pathways for inhibitory signaling. Cell 1997;90:293-301.

- 6 Hawkins PT, Anderson KE, Davidson K, Stephens LR: Signalling through class I PI3Ks in mammalian cells. Biochem Soc Trans 2006; 34:647-662.
7 Katso R, Okkenhaug K, Ahmadi K, White S, Timms J, Waterfield MD: Cellular function of phosphoinositide 3-kinases: implications for development, homeostasis, and cancer. Annu Rev Cell Dev Biol 2001;17:615-675.

8 Crabbe T, Welham MJ, Ward SG: The PI3K inhibitor arsenal: choose your weapon! Trends Biochem Sci 2007;32:450-456.

9 Rommel C, Camps M, Ji H: PI3K delta and PI3K gamma: partners in crime in inflammation in rheumatoid arthritis and beyond? Nat Rev Immunol 2007;7:191-201.

10 Vanhaesebroeck B, Guillermet-Guibert J, Graupera M, Bilanges B: The emerging mechanisms of isoform-specific PI3K signalling. Nat Rev Mol Cell Biol 2010;11:329341. DOI: $10.1038 / \mathrm{nrm} 2882$.

11 Mackay C: Moving targets: cell migration inhibitors as new anti-inflammatory therapies. Nat Immunol 2008;9:988-998.
12 Kerr WG, Heller M, Herzenberg LA: Analysis of lipopolysaccharide-response genes in B-lineage cells demonstrates that they can have differentiation stage-restricted expression and contain SH2 domains. Proc Natl Acad Sci USA 1996;93:3947-3952.

13 Damen JE, et al: The $145-\mathrm{kDa}$ protein induced to associate with Shc by multiple cytokines is an inositol tetraphosphate and phosphatidylinositol 3,4,5-triphosphate 5-phosphatase. Proc Natl Acad Sci USA 1996;93: 1689-1693.

14 Lioubin MN, et al: p150Ship, a signal transduction molecule with inositol polyphosphate-5-phosphatase activity. Genes Dev 1996;10:1084-1095.

15 Kavanaugh WM, et al: Multiple forms of an inositol polyphosphate 5-phosphatase form signaling complexes with Shc and Grb2. Curr Biol 1996;6:438-445.

16 Lucas DM, Rohrschneider LR: A novel spliced form of SH2-containing inositol phosphatase is expressed during myeloid development. Blood 1999;93:1922-1933. 
17 Ong CJ, et al: Small-molecule agonists of SHIP1 inhibit the phosphoinositide 3-kinase pathway in hematopoietic cells. Blood 2007; 110:1942-1949. DOI: 10.1182/blood-200703-079699.

18 Lamkin TD, et al: Shc interaction with Src homology 2 domain containing inositol phosphatase (SHIP) in vivo requires the Shcphosphotyrosine binding domain and two specific phosphotyrosines on SHIP. J Biol Chem 1997;272:10396-10401.

19 Huber M, et al: The src homology 2-containing inositol phosphatase (SHIP) is the gatekeeper of mast cell degranulation. Proc Natl Acad Sci USA 1998;95:11330-11335.

$\checkmark 20$ Verdier F, et al: Erythropoietin induces the tyrosine phosphorylation of insulin receptor substrate-2. An alternate pathway for erythropoietin-induced phosphatidylinositol 3-kinase activation. J Biol Chem 1997;272: 26173-26178.

-21 Zamorano J, Keegan AD: Regulation of apoptosis by tyrosine-containing domains of IL4R alpha: Y497 and Y713, but not the STAT6docking tyrosines, signal protection from apoptosis. J Immunol 1998;161:859-867.

22 Zhang S, Mantel C, Broxmeyer HE: Flt3 signaling involves tyrosyl-phosphorylation of SHP-2 and SHIP and their association with Grb2 and Shc in Baf3/Flt3 cells. J Leukoc Biol 1999;65:372-380.

23 Drachman JG, Kaushansky K: Dissecting the thrombopoietin receptor: functional elements of the Mpl cytoplasmic domain. Proc Natl Acad Sci USA 1997;94:2350-2355.

24 Ono M, Bolland S, Tempst P, Ravetch JV: Role of the inositol phosphatase SHIP in negative regulation of the immune system by the receptor Fc(gamma)RIIB. Nature 1996;383: 263-266.

25 Galandrini R, et al: SH2-containing inostiol phosphatase (SHIP-1) transiently translocates to raft domains and modulates CD16mediated cytotoxicity in human NK cells. Blood 2002;100:4581-4590.

26 Wang JW, et al: Influence of SHIP on the NK repertoire and allogeneic bone marrow transplantation. Science 2002;295:20942097.

27 Eissmann P, et al: Molecular basis for positive and negative signaling by the natural killer cell receptor 2B4 (CD244). Blood 2005; 105:4722-4729.

28 Abramson J, Pecht I: Clustering the mast cell function-associated antigen (MAFA) leads to tyrosine phosphorylation of p62Dok and SHIP and affects RBL-2H3 cell cycle. Immunol Lett 2002;82:23-28.

$29 \mathrm{Tu}$ Z, et al: Embryonic and hematopoietic stem cells express a novel $\mathrm{SH} 2$-containing inositol 5'-phosphatase isoform that partners with the Grb2 adapter protein. Blood 2001;98:2028-2038.

30 Damen JE, et al: The role of erythropoietin receptor tyrosine phosphorylation in erythropoietin-induced proliferation. Leukemia 1997;11:423-425.
Kalesnikoff J, et al: SHIP negatively regulates IgE + antigen-induced IL- 6 production in mast cells by inhibiting NF-kappa B activity. J Immunol 2002;168:4737-4746.

32 Strassheim D, Kim JY, Park JS, Mitra S, Abraham E: Involvement of SHIP in TLR2induced neutrophil activation and acute lung injury. J Immunol 2005;174:8064-8071.

33 Robson JD, Davidson D, Veillette A: Inhibition of the Jun $\mathrm{N}$-terminal protein kinase pathway by SHIP-1, a lipid phosphatase that interacts with the adaptor molecule Dok-3. Mol Cell Biol 2004;24:2332-2343.

34 Kiessling R, et al: Killer cells: a functional comparison between natural, immune T-cell and antibody-dependent in vitro systems. J Exp Med 1976;143:772-780.

35 Haller O, Wigzell H: Suppression of natural killer cell activity with radioactive strontium: effector cells are marrow dependent. J Immunol 1977;118:1503-1506.

36 Ojo E, Wigzell H: Natural killer cells may be the only cells in normal mouse lymphoid cell populations endowed with cytolytic ability for antibody-coated tumour target cells. Scand J Immunol 1978;7:297-306.

37 Kanakaraj P, et al: Phosphatidylinositol-3 kinase activation induced upon Fc gamma RIIIA-ligand interaction. J Exp Med 1994;179: 551-558.

- 38 Bonnema JD, Karnitz LM, Schoon RA, Abraham RT, Leibson PJ: Fc receptor stimulation of phosphatidylinositol 3-kinase in natural killer cells is associated with protein kinase C-independent granule release and cell-mediated cytotoxicity. J Exp Med 1994; 180:1427-1435.

39 Jiang K, et al: Pivotal role of phosphoinositide- 3 kinase in regulation of cytotoxicity in natural killer cells. Nat Immunol 2000;1: 419-425. DOI: $10.1038 / 80859$.

40 Colucci F, et al: Natural cytotoxicity uncoupled from the Syk and ZAP-70 intracellular kinases. Nat Immunol 2002;3:288-294. DOI: 10.1038/ni764.

41 Zompi S, et al: NKG2D triggers cytotoxicity in mouse NK cells lacking DAP12 or Syk family kinases. Nat Immunol 2003;4:565572.

42 Fruman DA, et al: Impaired B cell development and proliferation in absence of phosphoinositide 3-kinase p85alpha. Science 1999;283:393-397.

43 Suzuki H, et al: Xid-like immunodeficiency in mice with disruption of the p85alpha subunit of phosphoinositide 3-kinase. Science 1999;283:390-392.

44 Saudemont A, Okkenhaug K, Colucci F: p110delta is required for innate immunity to transplantable lymphomas. Biochem Soc Trans 2007;35: 183-185. DOI: $\underline{10.1042 /}$ BST0350183.

45 Kim N, et al: The p110delta catalytic isoform of PI3K is a key player in NK-cell development and cytokine secretion. Blood 2007; 110:3202-3208. DOI: 10.1182/blood-2007$\underline{02-075366}$
46 Saudemont A, et al: p110gamma and p110delta isoforms of phosphoinositide 3-kinase differentially regulate natural killer cell migration in health and disease. Proc Natl Acad Sci USA 2009;106:5795-5800. DOI: 10.1073/ pnas0808594106.

47 Tassi I, et al: p110gamma and p110delta phosphoinositide 3-kinase signaling pathways synergize to control development and functions of murine NK cells. Immunity 2007;27:214-227.

48 Guo H, Samarakoon A, Vanhaesebroeck B, Malarkannan S: The p110 delta of PI3K plays a critical role in NK cell terminal maturation and cytokine/chemokine generation. J Exp Med 2008;205:2419-2435. DOI: 10.1084/ jem20072327.

49 Zebedin E, et al: Leukemic challenge unmasks a requirement for PI3Kdelta in NK cell-mediated tumor surveillance. Blood 2008;112:4655-4664. DOI: 10.1182/blood2008-02-139105.

50 Orr MT, Lanier LL: Natural killer cell education and tolerance. Cell 2010;142:847-856. DOI: $10.1016 /$ jcell201008031.

51 Hoglund P, Brodin P: Current perspectives of natural killer cell education by MHC class I molecules. Nat Rev Immunol 2010;10:724734. DOI: $10.1038 /$ nri2835.

52 Kim S, et al: Licensing of natural killer cells by host major histocompatibility complex class I molecules. Nature 2005;436:709-713.

53 Orr MT, Murphy WJ, Lanier LL: 'Unlicensed' natural killer cells dominate the response to cytomegalovirus infection. Nat Immunol 2010;11:321-327. DOI: 10.1038/ni1849.

54 Carrega P, et al: Susceptibility of human melanoma cells to autologous natural killer (NK) cell killing: HLA-related effector mechanisms and role of unlicensed NK cells. PLoS One 2009;4:e8132. DOI: 10.1371/journalpone 0008132

$55 \overline{\text { Tay CH, Welsh R }}$, Brutkiewicz RR: NK cell response to viral infections in beta 2-microglobulin-deficient mice. J Immunol 1995; 154:780-789.

56 Fortenbery NR, et al: SHIP influences signals from CD48 and MHC class I ligands that regulate NK cell homeostasis, effector function, and repertoire formation. J Immunol 2010;184:5065-5074. DOI: 10.4049/jimmunol0901862.

57 Wahle JA, et al: Cutting edge: dominance by an MHC-independent inhibitory receptor compromises NK killing of complex targets. J Immunol 2006;176:7165-7169.

58 Wahle JA, et al: Inappropriate recruitment and activity by the Src homology region 2 domain-containing phosphatase 1 (SHP1) is responsible for receptor dominance in the SHIP-deficient NK cell. J Immunol 2007; 179: 8009-8015.

59 Kishimoto $\mathrm{H}$, et al: The Pten/PI3K pathway governs the homeostasis of Valpha14iNKT cells. Blood 2007; 109:3316-3324. DOI: 10.1182/blood-2006-07-038059. 
60 Desponts C, Ninos JM, Kerr WG: s-SHIP associates with receptor complexes essential for pluripotent stem cell growth and survival. Stem Cells Dev 2006;15:641-646.

61 Lowin-Kropf B, Held W: Positive impact of inhibitory Ly49 receptor-MHC class I interaction on NK cell development. J Immunol 2000;165:91-95.

62 Kerr WG: Acquisition and remodeling of the NK receptor repertoire; in Brossay L (ed): Everything You Wanted to Know About NK Cells, But Were Afraid to Ask. Kerala, Research Signpost, 2007.

63 Marti F, et al: LCK-phosphorylated human killer cell-inhibitory receptors recruit and activate phosphatidylinositol 3-kinase. Proc Natl Acad Sci USA 1998;95:11810-11815.

-64 McVicar DW, et al: Aberrant DAP12 signaling in the 129 strain of mice: implications for the analysis of gene-targeted mice. J Immunol 2002;169:1721-1728.

-65 Johansson S, et al: Natural killer cell education in mice with single or multiple major histocompatibility complex class I molecules. J Exp Med 2005;201:1145-1155.
66 Khoo NK, Fahlen L, Sentman CL: Modulation of Ly49A receptors on mature cells to changes in major histocompatibility complex class I molecules. Immunology 1998;95: 126-131.

$67 \mathrm{Kim} \mathrm{CH}$, et al: Altered responsiveness to chemokines due to targeted disruption of SHIP. J Clin Invest 1999;104:1751-1759.

68 Hazen AL, et al: SHIP is required for a functional hematopoietic stem cell niche. Blood 2009;113:2924-2933. DOI: 10.1182/blood2008-02-138008.

69 Desponts C, Hazen AL, Paraiso KH, Kerr WG: SHIP deficiency enhances HSC proliferation and survival but compromises homing and repopulation. Blood 2006;107:43384345.

70 Nishio M, et al: Control of cell polarity and motility by the PtdIns $(3,4,5) \mathrm{P}_{3}$ phosphatase SHIP1. Nat Cell Biol 2007;9:36-44. DOI: 10.1038/ncb1515.

71 Trotta R, et al: Differential expression of SHIP1 in CD56 $6^{\text {bright }}$ and CD56 ${ }^{\text {dim }}$ NK cells provides a molecular basis for distinct functional responses to monokine costimulation. Blood 2005;105:3011-3018.
72 Kerr WG, Park MY, Maubert M, Engelman RW: SHIP deficiency causes Crohn's disease-like ileitis. Gut 2011;60:177-188. DOI: 10.1136/gut2009202283.

73 Cella M, et al: A human natural killer cell subset provides an innate source of IL-22 for mucosal immunity. Nature 2009;457:722725. DOI: $10.1038 /$ nature 07537.

74 Satoh-Takayama N, et al: Microbial flora drives interleukin 22 production in intestinal NKp46+ cells that provide innate mucosal immune defense. Immunity 2008;29: 958-970. DOI: 10.1016/jimmuni200811001.

75 Sanos SL, et al: RORgammat and commensal microflora are required for the differentiation of mucosal interleukin 22-producing NKp46+ cells. Nat Immunol 2009;10:83-91. DOI: 10.1038/ni.1684.

76 Zenewicz LA, et al: Innate and adaptive interleukin-22 protects mice from inflammatory bowel disease. Immunity 2008;29:947957. DOI: $10.1016 /$ jimmuni200811003. 